\title{
In Situ Localization of AOS in Host-Pathogen Interactions
}

\author{
Klaus B. Tenberge , ${ }^{*}$ Marcus Beckedorf,* Britta Hoppe, ${ }^{*}$ Alexander Schouten, ${ }^{* *}$ Martina Solf, ${ }^{*}$ \\ and Marcell von den Driesch*
* Institut für Botanik, Westfälische Wilhelms-Universität Münster, Schlossgarten 3, D-48149 Münster, Germany; email: tenberg@uni-muenster.de
** Wageningen University Plant Sciences, Laboratory of Phytopathology, PO Box 8025, 6700 EE Wageningen, The Netherlands

Active oxygen species (AOS) of host origin accumulate transiently in plant apoplasts in response to pathogen attack. This rapid reaction is called oxidative burst and probably functions in defense reactions directly or in signaling [1]. The aim of the presented work is to gain insight in role and source of AOS by visualizing the spatio-temporal occurrence of AOS during pathogenesis. As the role of AOS seems to vary with the life strategy of a pathogen, we investigate the interactions of a necrotrophic and of a biotrophic pathogen with their respective hosts.

Botrytis cinerea causes serious diseases, called grey mould, in at least 235 plant species, including important crops. The pathogen is a typical necrotroph, inducing host cell death before invasion. As the oxidative burst usually results in host cell death, the pathogen might utilize this induced suicide during the preparation of its primary infection court. In addition, the pathogen might produce AOS itself to intensify the effect.

The histopathology was investigated using light and electron microscopy. Spore adhesion, germination and swellings of germ tube tips attached to the surface by a matrix material was documented on tomato and bean. Germ tube tip swellings were demonstrated to develop functional appressoria that mediated cuticle penetration and infection hypha formation in the outer epidermal wall underneath (Fig. 1). Using LM, chloronaphthol and NBT staining were applied to visualize hydrogen peroxide and superoxide, respectively. The primary lesion area stained positive for AOS and most intense at its border zone. With time this concentric zone spread in the leaf mesophyll. The source of this wavelike AOS production was the host oxidative burst. This was visualized even by LM at higher magnification and further substantiated in TEM. The cerium chloride technique specific for hydrogen peroxide resulted in electron dense precipitate of cerium perhydroxide at the interface of $B$. cinerea and host cells [2]. This precipitate was present in the periplasmic space, in the host cell wall and at the outer surface of the host cell as well as at the outside of the fungal wall. Consequently, $\mathrm{H}_{2} \mathrm{O}_{2}$ was produced in host cells at the plasma membrane and diffuses through the host cell wall into the intercellular space.

Rectangular crystals were found to be an interaction-specific reaction product on lesion surfaces. Cytochemistry and ESEM with EDX revealed that they were formed of calcium oxalate. This could serve as substrate of fungal oxalate oxidases generating hydrogen peroxide. Notably, other cytological and biochemical evidence established the presence of an AOS secretion pathway in the pathogen. AOS specific staining was observed inside and outside of vital fungal cells in axenic culture and during pathogenesis. Activity staining and use of specific inhibitors substantiated that fungal superoxide dismutases were involved in this process. Using TEM, secreted hydrogen peroxide was visualized during penetration, indicating an oxidative attack upon the host cuticle and outer epidermal cell wall (Fig. 2). This seems to be a new and important discovery, since target mutagenesis ruled out an essential role of a cutinase gene in the pathogenesis of grey mould [3].

Claviceps purpurea causes ergot disease by infecting ovaries of grasses. This pathogen is a holobiotroph which obtains nutrients only from living host tissue while managing to maintain host cell viability for extended periods. Since $C$. purpurea produces compatible interactions with hundreds of host species, we hypothesized that the fungus might interfere with $\mathrm{H}_{2} \mathrm{O}_{2}$-mediated 
defense by means of secreted catalases and other AOS scavenging enzymes [4, 5, 6]. While investigating the spatio-temporal distribution of different AOS and related enzymes in this system, cytochemical evidence indicated that this pathogen also produces AOS itself [7].

\section{References}

[1] C.S. Bestwick et al., Plant Cell 9 (1997) 209-221.

[2] T.W. Prins et al., in Fungal Pathology, pp. 33-63, Kronstad J.W., ed., Kluwer Academic Publishers, Dordrecht, 2000.

[3] J.A.L. van Kan et al., Mol. Plant-Microbe Interact. 10 (1997) 30-38.

[4] V. Garre et al., Phytopathology 88 (1998) 744-753.

[5] V. Garre et al., Mol. Plant-Microbe Interact. 11 (1998) 772-783.

[6] P. Tudzynski and K.B. Tenberge, in Clavicipitalean Fungi: Evolutionary Biology, Chemistry, Biocontrol and Cultural Impacts, J.F. White et al., eds., Marcel Dekker, Inc., New York (in press).

[7] Our research was funded by the EU in the EU-FAIR project and by the DFG.
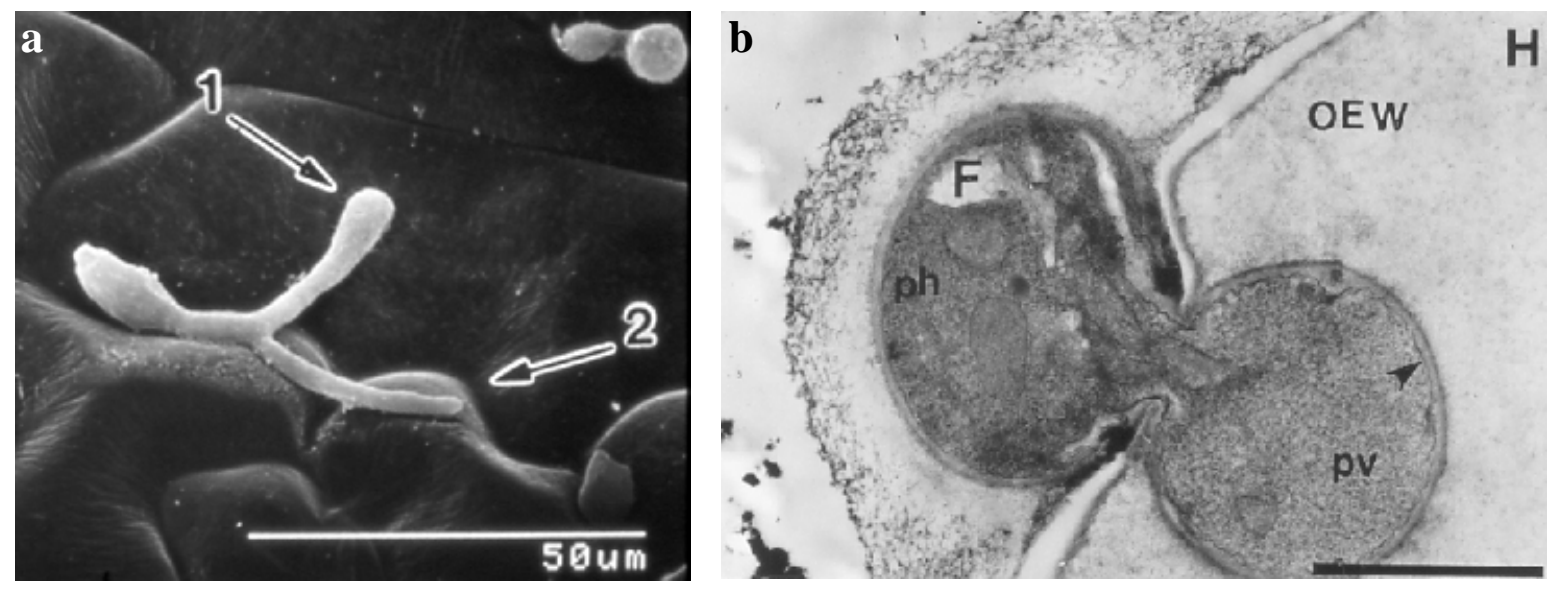

Fig. 1. Infection structures of Botrytis on bean leaves. a, A tip swelling (1) of a germ tube. $\mathrm{b}$, Appressorium-mediated penetration of $B$. cinerea $(\mathrm{F})$ into the outer epidermal wall $(\mathrm{OEW})$ of a bean leaf $(\mathrm{H})$ at $12 \mathrm{hpi}$. Scale bar, $1 \mu \mathrm{m}$.
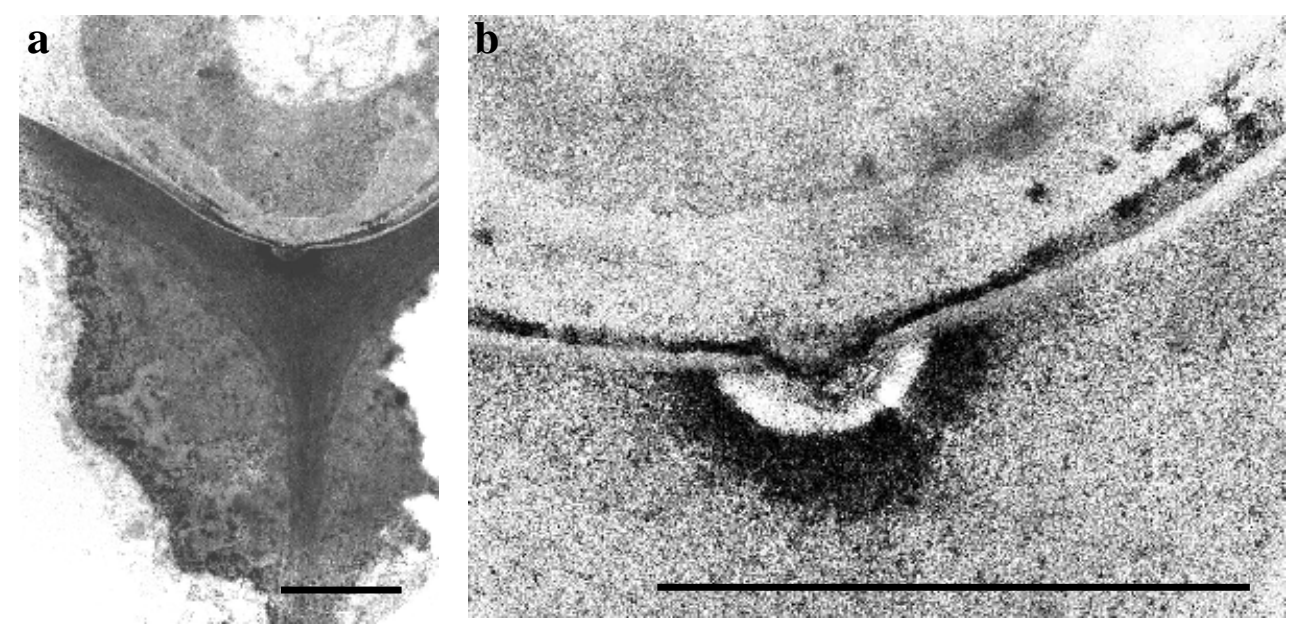

Fig. 2: Localization of $\mathrm{H}_{2} \mathrm{O}_{2}$ by cerium chloride at the interface of a $B$. cinerea appressorium and the outer epidermal wall of a tomato leaf at 12 hpi. Overview (a) and detail (b) from a section series. 